\title{
Similarity in Predictors between Near Miss and Adverse Event among Japanese Nurses Working at Teaching Hospitals
}

\author{
Katsutoshi TANAKA ${ }^{1 *}$, Tempei OTSUBO ${ }^{2}$, Mika TANAKA ${ }^{3}$, Akiko KAKU ${ }^{1}$, \\ Nao NISHINOUE ${ }^{1}$, Tomoki TAKANAO ${ }^{1}$, Naoki KAMATA ${ }^{4}$ and Hitoshi MIYAOKA ${ }^{4}$ \\ ${ }^{1}$ Department of Occupational Mental Health, Graduate School of Medical Sciences, Kitasato University, 1-15-1 \\ Kitasato, Sagamihara, Kanagawa 228-8555, Japan \\ ${ }^{2}$ Department of Psychiatry, Tokyo Women's Medical University School of Medicine, 8-1 Kawada-cho, \\ Shinjuku-ku, Tokyo 162-8666, Japan \\ ${ }^{3}$ School of Nursing, Faculty of Medicine, Fukuoka University, 7-45-1 Nanakuma, Jonan-ku, Fukuoka \\ 814-0180, Japan \\ ${ }^{4}$ Department of Psychiatry, Kitasato University School of Medicine, 1-15-1 Kitasato, Sagamihara, Kanagawa \\ 228-8555, Japan
}

Received November 1, 2009 and accepted December 25, 2009

\begin{abstract}
Near miss-based analysis has been recently suggested to be more important in the medical field than focusing on adverse events, as in the industrial field. To validate the utility of near miss-based analysis in the medical fields, we investigated whether or not predictors of near misses and adverse events were similar among nurses at teaching hospitals. Of the 1,860 nurses approached, 1,737 $(93.4 \%)$ were included in the final analysis. Potential predictors provided for analysis included gender, age, years of nursing experience, frequency of alcohol consumption, work place, ward rotation, frequency of night shifts, sleepiness during work, frequency of feeling unskilled, nurses' job stressors, working conditions, and depression. Variables for multivariate analysis were determined by bivariable analysis. Ordinal logistic analysis showed that predictors of near misses and adverse events were markedly similar. Parameters that were significantly related to both near misses and adverse events were years of experience, frequency of night shifts, internal ward, and time pressure $(p<0.05$ for all). The present study suggested that there was a negligible difference between choosing near miss- or adverse eventbased analysis when identifying possible causes of adverse events in the medical field.
\end{abstract}

Key words: Predictor, Near miss, Adverse event, Nurse, Prospective cohort study

\section{Introduction}

Patient safety improvement is a high-priority issue of social import in many countries. While several previous studies have reported on the large number of people who are injured or die from medical errors, stricter countermeasures are yet needed to prevent error-induced patient harm and improve patient safety ${ }^{1-3)}$.

Several terms have been brought into use to define

*To whom correspondence should be addressed.

E-mail: k-tanaka@kitasato-u.ac.jp outcomes occurring due to error in the medical field, with the two primary categories being "near misses" (also called as close misses or potential adverse events) and "adverse events" (also known as accidents or harmful events $)^{4)}$.

In industrial fields such as air and rail transport or nuclear power and chemical plants, systems have been developed to prevent unexpected accidents, involving voluntarily reporting and analyzing near misses (the term "accident" is typically used instead of "adverse event" in industrial fields) $)^{5)}$. Heinrich's report ${ }^{6)}$, which is often cited in the prevention of industrial accidents, 
indicates that near miss-based analysis and countermeasures against near misses are essential for implementing effective accident prevention. Such an approach emphasizing near misses is based on the concept that predictors of accidents and near misses are basically the same ${ }^{7,8)}$, and greater attention may be focused on near misses simply due to the fact that accidents occur much less frequently than near misses.

In the medical field, adverse-event studies, especially those that investigate frequency of deaths or serious injuries, have been performed. In most of these studies, occurrences of adverse events were detected by direct observation. Recent studies have reported that voluntary reporting provide more useful information regarding errors and their causes than information provided by direct observation and mandatory reporting ${ }^{9,10)}$. It has more recently been considered that focusing on near misses is more important in the medical field than focusing on adverse events, as in the industrial

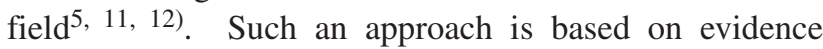
that near misses can be quantitatively analyzed given the abundant data available, the fact that resisters to data collection for near misses are fewer then for adverse events $\left.{ }^{13}, 14\right)$, and based on the hypothesis that the predictors of adverse events and near misses are similar.

To implement this approach, however, we must first ascertain that predictors of near misses and adverse events are indeed similar in the medical field. Most studies performed in the medical field thus far have evaluated the outcomes of either adverse events or near misses, and none have determined whether or not predictors of near misses and adverse events can strictly be considered similar ${ }^{5}$. Conditions differ between the medical and industrial fields; for example, certain preventive measures are more advanced in the industrial field, and frequency of adverse events (or "accidents") is actually much higher in the medical field.

The immediate purpose of the error analysis is to prevent adverse events. If a significant difference is observed in predictors between near miss and adverse event analysis, we must practice caution when emphasizing near miss analysis in the medical field. In this prospective study, in order to validate the utility of near miss-based analysis when identifying possible causes of adverse events in the medical field, we investigated whether or not predictors of near misses and adverse events were similar among nurses at teaching hospitals.

\section{Methods}

\section{Participants and procedures}

This study enrolled 1,860 registered nurses working at 5 teaching hospitals in Tokyo and Kanagawa, Japan.
Among the five hospitals, two hospitals (526 nurses and 353 nurses) had the same type of three-shift system that involve 8-h night work, and three hospitals (686 nurses, 161 nurses and 134 nurses) had the same type of twoshift system that involve 16-h night work. Hospital climate with regard to safety and principles for medical error prevention was similar between hospitals. The five hospitals are teaching institutions and share safety provisions such as providing incident management systems and on-the-job education programs for incident prevention.

Hospital wards and outpatient departments are staffed by a senior nursing officer and one or two chief nurses. Senior nursing officers were excluded from the study because they were in charge of staff management and were not primarily involved in nursing care. Chief nurses were included in the study because they provided nursing care in addition to their management jobs. No other exclusion criteria were specified.

The objectives and procedures of this study were explained to the nursing director and senior nursing officer at each hospital, and the following information was provided to the nurses using a study information sheet: objectives and methods of the study, and notice of voluntary participation, no disadvantages of nonparticipation, an option to discontinue the study at any point. Informed consent was given on returning a selfreport questionnaire to the investigator.

The first self-report questionnaire was distributed to all nurses at five hospitals in November 2005. To link the first and second surveys, participants were instructed in the initial questionnaire to provide a self-defined four-digit ID number and a hint, should the ID number be forgotten, such as the name of the participant's junior high school. The questionnaire also inquired about the following potential predictors that have been reported to be significantly associated with medical errors ${ }^{15-22)}$ : gender, age, years of nursing experience, number of drinking days per week, work place, presence or absence of a ward rotation in the previous six months, frequency of night shifts, frequency of sleepiness during work regardless of day or night shifts, highest severity of sleepiness assessed on the Stanford sleepiness scale during a day shift, frequency of feeling unskilled, job stressors in nursing, working conditions, and feelings of depression. Response choices regarding frequency of feeling sleepy during work or feeling unskilled were "no", "occasionally", "frequently", or "always". In the analysis, responses of "no" and "occasionally" were regarded as "no", while "frequently" and "always" were regarded as "yes".

The second questionnaire, distributed in May 2006, inquired about the participant's ID number or hint and 
the frequency of self-perceived near misses and adverse events experienced in the last six months. The participants had been instructed ahead of time to assess and record the number of near misses and adverse events during observation period. All reported questionnaires were sealed in individual envelopes before collection.

Approval to conduct the study was obtained from the Institutional Ethics Committees of Showa and Kitasato Universities.

\section{Measurements}

Definition of "near miss" and "adverse event"

In the medical field, a near miss is simply categorized as an error resulting in no harm to the patient ${ }^{18)}$. In the present study, an error-related near miss was defined as an unanticipated incident in which an error was made but no harm occurred, and frequency was inquired about in the questionnaire as, "In the previous six months, how often did you experience a self-errorrelated near miss in which an error was made but no harm occurred to a patient?'

An adverse event is generally defined as an injury related to medical management ${ }^{23)}$. An adverse event caused by an error is known as a "preventable adverse event", to distinguish it from an unpreventable event such as an adverse reaction to an unknown drug $^{24)}$. In the present study, an adverse event was limited to a preventable adverse event and was defined as an unanticipated incident in which an error was made and harm occurred. Frequency of perceived adverse events in the previous six months was inquired about in the questionnaire as, "In the previous six months, how often did you inflict a self-error-related injury on a patient or disadvantage a patient, regardless of event severity?"

Assessment of job stressors in nursing, working conditions, and depression

Nursing-specific job stressors were evaluated using the well-known and widely implemented Nursing Stress Scale (NSS ${ }^{25-27)}$. Sources of stressors are comprised of seven subscales: death and dying, conflict with physicians, inadequate preparation, problems with peers and supervisors, workload, and uncertainty concerning treatment. In the present study, total NSS scores were used for analyses.

Working condition regarded as related to medical errors $16,17,19,20,22)$ was inquired about in the questionnaire as, "How often do you feel strong time pressure during work?" and, "How often do you feel a lack of communication among staff members, including all medical doctors and workers?" Response choices were "no", "occasionally", "frequently", or "always". In the analysis, responses of "no" and "occasionally" were regarded as "no", while "frequently" and "always" were regarded as "yes".

The nurse's workplace was either the outpatient department, internal ward, surgical ward, or emergency room/intensive care unit (ER/ICU). The internal ward included internal medicine, dermatology, neurology, and rehabilitation. The surgical ward included pediatrics, obstetrics, gynecology, ophthalmology, urology, otorhinology, anesthesiology (often many patients hospitalized for surgery in these wards), and all surgical departments (cardiovascular, gastrointestinal, respiratory, cerebral, orthopedic, and plastic surgery). The ER/ICU included the pediatric and neonatal intensive care units and the operating room.

Nurse depression was assessed using the Hospital Anxiety and Depression Scale (HADS) ${ }^{28}$. The HADS is a short self-report questionnaire comprising two subscales for anxiety and depression, each with a score between 0 and 21 . While both the validity and cut-off point of the English-language version of the HADS have been previously evaluated ${ }^{29}$ ), the validity of the cutoff point has not been studied in the Japanese-language version. Therefore, the depression scores obtained from HADS in the present study were used directly.

\section{Statistical analyses}

A nurse who experienced both a near miss and an adverse event in the same observation period was regarded as having experienced an adverse event and were thus excluded from the near miss analysis. Therefore, the frequency of near misses was ultimately defined as the frequency of near misses reported by nurses who did not also experience adverse events. Given that frequencies of near misses and adverse events were not commonly normally distributed, we divided the frequency of near misses and adverse events experienced in the previous six months into tertiles before analysis.

Variables for multivariate analysis were determined by bivariable analysis with $p$ value of less than 0.05 . Categorical variables were analyzed by Fisher's exact test, and continuous variables by Dunnett's multiple comparison test. The tertiles of near misses and adverse events were used as ordinal dependent variables, and relationships with potential predictors were analyzed using ordinal logistic regression analysis with the generalized estimating equation with hospitals as the random effects. Akaike's information criterion (AIC) was selected for the final model. SPSS version 16.0 (SPSS Inc., Chicago, IL, USA) was used for analyses, and statistical significance was set at 0.05 , with all tests two-tailed. 


\section{Results}

Baseline self-report questionnaires were obtained from 1,815 of 1,860 nurses $(97.6 \%)$, with no information available regarding the 45 nurses who did not respond to the baseline questionnaire. Of these 1,815 who responded to the baseline questionnaire, $1,788(96.1 \%)$ responded to the second questionnaire. Of these 1,788 who responded to both questionnaires, the 1,737 (93.4\%) who responded to questions on gender, age, years of nursing experience, alcohol consumption, frequency of night shifts, working conditions, nursing stressors, depression, and frequency of perceived near misses and adverse events were ultimately included in statistical analyses. No significant difference was noted for gender, age, years of nursing experience, alcohol consumption, frequency of night shifts, working conditions, NSS scores, or depression scores between the 78 nurses who responded only to the baseline questionnaire or poorly responded to the second and the 1,737 nurses included in the final analysis.

Demographic characteristics of the study nurses are shown in Table 1. Of the 1,737 nurses included in the final analysis, 1,649 (94.9\%) were female, with a mean (SD) age of 29.0 (6.7) $\mathrm{yr}$ and 6.8 (6.2) mean years of experience. A total of 1,407 nurses $(81.0 \%)$ worked on a rotation schedule including a nightshift. Of the 1,737 nurses, 865 (49.8\%) experienced no adverse events during the 6-month observation period. These 865 nurses comprised 199 (23.0\%) who experience no near misses, 280 (32.4\%) who experienced 1 near miss, and 386 $(44.6 \%)$ who experienced 2 or more near misses. The mean (SE) frequency of near misses for these 865 nurses was $1.85(0.07)$. The calculated frequency of near misses was zero in the lowest tertile, one in the middle tertile, and two-or-more in the highest tertile.

The 1,737 nurses comprised $865(49.8 \%)$ who experienced no adverse events, 441 (25.4\%) who experienced 1 event, and $431(24.8 \%)$ who experienced 2 or more events in the previous 6 months. The mean frequency (SE) of adverse events for six months was $1.00(0.03)$. The calculated frequency of adverse events was zero in the lowest tertile, one in the middle tertile, and two-ormore in the highest tertile.

The results of bivariable analysis are shown in Table 2 . The parameters significantly related to near misses were age, years of experience, workplace, frequency of night shifts, lack of skill, time pressure, a lack of communication between medical staff, nursing stressors, and depression. The parameters significantly related to adverse events were the same as those for near misses, except for depression. No significant relationship

Table 1. Demographic characteristics of study participants $(\mathrm{N}=1,737)$

\begin{tabular}{lc}
\hline Number of females & $1,649(94.9 \%)$ \\
Age in years, mean (SD) & $29.0(6.7)$ \\
Years of nursing experience, mean (SD) & $6.8(6.2)$ \\
Number of drinking days per week, mean (SD) & $0.7(1.0)$ \\
Workplace & \\
$\quad$ Outpatient department & $221(12.8 \%)$ \\
$\quad$ Internal ward & $730(42.3 \%)$ \\
$\quad$ Surgical ward & $461(26.7 \%)$ \\
$\quad$ ER or ICU & $313(18.1 \%)$ \\
Were on a ward rotation in the last six months & $257(14.8 \%)$ \\
Were on a night shift & $1,407(81.0 \%)$ \\
Number of night shifts per month, mean (SD) & $5.0(3.5)$ \\
Severity of sleepiness during the day work, mean (SD) & $2.8(1.0)$ \\
Frequently experience sleepiness during work & 25 \\
Lack the skills) & $350(20.3 \%)$ \\
Experience time pressure ${ }^{2)}$ & $690(39.9 \%)$ \\
Lack of communication ${ }^{2)}$ & $426(24.6 \%)$ \\
Nnursing stress, mean (SD) ${ }^{3)}$ & $1,086(62.7 \%)$ \\
Depression, mean (SD) & $37.7(12.7)$ \\
\hline ER, emergency room; ICU, intensive care unity; SD, standard deviation. & \\
1) Assessed using the Stanford Sleepiness Scale. & $7.5(4.1)$ \\
2)Answered with "no", "occasionally", "frequently", or “always". "No" and \\
"occasionally" were regarded as no, and "frequently" and "always" were regard- \\
ed as yes. \\
3)Assesessed using the Nursing Stress Scale. \\
4)Assesessed using the Hospital Anxiety and Depression Scale.
\end{tabular}


was observed between frequencies of near misses and adverse events and gender, number of drinking days per week, recent ward rotation, and severity of sleepiness and frequency of feeling sleepy during work.

The results of ordinal logistic regression analysis with dependent variables for near misses and adverse events are shown in Table 3. Variables found to be significantly related to a near miss or adverse event by bivariable analysis were used as potential predictors. To adjust the effects of hospitals, the generalized estimating

Table 2. Association of near misses and adverse events with demographic characteristics

\begin{tabular}{|c|c|c|c|c|c|c|c|c|}
\hline & \multicolumn{4}{|c|}{ Frequencies of near misses } & \multicolumn{4}{|c|}{ Frequencies of adverse events } \\
\hline & $\begin{array}{c}0 \\
(\mathrm{~N}=199)\end{array}$ & $\begin{array}{c}1 \\
(\mathrm{~N}=280)\end{array}$ & $\begin{array}{c}2+ \\
(\mathrm{N}=386)\end{array}$ & $p$ value & $\begin{array}{c}0 \\
(\mathrm{~N}=865)\end{array}$ & $\begin{array}{c}1 \\
(\mathrm{~N}=441)\end{array}$ & $\begin{array}{c}2+ \\
(\mathrm{N}=431)\end{array}$ & $p$ value \\
\hline Number of females, No. (\%) & $193(97.0 \%)$ & $268(95.7 \%)$ & $364(94.3 \%)$ & 0.32 & $825(95.4 \%)$ & $421(95.5 \%)$ & $403(93.5 \%)$ & 0.30 \\
\hline Age in years, mean $(\mathrm{SD})$ & $32.0(7.0)$ & $30.2(7.0)$ & $29.5(6.8)$ & $<0.001$ & $30.3(7.0)$ & $28.0(5.9)$ & $27.2(6.2)$ & $<0.001$ \\
\hline Years of nursing experience, mean (SD) & $9.6(6.9)$ & $8.4(7.0)$ & $7.0(6.0)$ & $<0.001$ & $8.1(6.6)$ & $6.0(5.3)$ & $4.9(5.4)$ & $<0.001$ \\
\hline Number of drinking days per week, mean (SD) & $0.7(1.0)$ & $0.8(1.0)$ & $0.7(1.0)$ & 0.50 & $0.8(1.0)$ & $0.8(1.0)$ & $0.7(1.0)$ & 0.48 \\
\hline \multicolumn{9}{|l|}{ Workplace } \\
\hline Outpatient department, No. (\%) & $72(36.2 \%)$ & $44(15.7)$ & $47(12.2 \%)$ & $<0.001$ & $163(18.9 \%)$ & $37(8.5 \%)$ & $21(4.9 \%)$ & $<0.001$ \\
\hline Internal ward, No. (\%) & $60(30.2 \%)$ & $88(31.4 \%)$ & $175(45.6 \%)$ & & $323(37.4 \%)$ & $202(46.5 \%)$ & $205(47.9 \%)$ & \\
\hline Surgical ward, No. (\%) & $34(17.1 \%)$ & $92(32.9 \%)$ & $93(24.2 \%)$ & & $219(25.4 \%)$ & $103(23.7 \%)$ & $139(32.5 \%)$ & \\
\hline ER or ICU, No. (\%) & $33(16.6 \%)$ & $56(20.0 \%)$ & $69(18.0 \%)$ & & $158(18.3 \%)$ & $92(21.2 \%)$ & $63(14.7 \%)$ & \\
\hline Were on a ward rotation in the last six months, No. (\%) & $41(20.6 \%)$ & $43(15.4 \%)$ & $57(14.8 \%)$ & 0.17 & $141(16.3 \%)$ & $60(13.7 \%)$ & $56(13.1 \%)$ & 0.22 \\
\hline Number of night shifts per month, mean (SD) & $2.8(3.1)$ & $4.6(3.4)$ & $5.0(3.3)$ & $<0.001$ & $4.4(3.4)$ & $5.4(3.3)$ & $5.9(3.4)$ & $<0.001$ \\
\hline Severity of sleepiness, mean (SD) ${ }^{1)}$ & $2.7(1.1)$ & $2.8(1.0)$ & $2.8(1.1)$ & 0.21 & $2.8(1.1)$ & $2.9(1.0)$ & $2.8(1.0)$ & 0.49 \\
\hline Frequency of sleepiness during work, No. $(\%)^{2)}$ & $34(17.1 \%)$ & $52(18.6 \%)$ & $87(22.6 \%)$ & 0.22 & $173(20.0 \%)$ & $89(20.2 \%)$ & $88(20.4 \%)$ & 0.99 \\
\hline Lack of skill, No. $(\%)^{2)}$ & $88(44.2 \%)$ & $142(50.7 \%)$ & $236(61.5 \%)$ & $<0.001$ & $466(54.0 \%)$ & $269(61.0 \%)$ & $303(71.5 \%)$ & $<0.001$ \\
\hline Time pressure, No. $(\%)^{2)}$ & $127(63.8 \%)$ & $201(71.8 \%)$ & $290(75.1 \%)$ & 0.02 & $618(71.4 \%)$ & $351(80.1 \%)$ & $339(78.7 \%)$ & $<0.01$ \\
\hline Lack of communication, No. $(\%)^{2)}$ & $51(25.6 \%)$ & $80(28.7 \%)$ & $155(40.4 \%)$ & $<0.001$ & $286(33.2 \%)$ & $162(36.8 \%)$ & $198(46.0 \%)$ & $<0.001$ \\
\hline Nursing stress, mean (SD) ${ }^{3)}$ & $31.7(12.4)$ & $35.0(13.0)$ & $39.6(12.6)$ & $<0.001$ & $36.3(13.1)$ & $38.4(11.3)$ & $39.7(12.9)$ & $<0.001$ \\
\hline Depression, mean $(\mathrm{SD})^{4)}$ & $6.0(3.5)$ & $6.8(3.9)$ & $8.4(3.9)$ & $<0.001$ & $7.3(4.0)$ & $7.6(4.4)$ & $7.9(4.1)$ & 0.08 \\
\hline
\end{tabular}

ER, emergency room; ICU, intensive care unity; SD, standard deviation.

1) Assessed using the Stanford Sleepiness Scale.

2) Answered with "no", "occasionally", "frequently", or "always". "No" and "occasionally" were regarded as no, and "frequently" and "always" were regarded as yes.

${ }^{3}$ Assesessed using the Nursing Stress Scale.

${ }^{4)}$ Assesessed using the Hospital Anxiety and Depression Scale.

Table 3. Difference in predictors between near misses and adverse events using multivariate analysis

\begin{tabular}{|c|c|c|c|c|}
\hline & \multicolumn{2}{|l|}{ Near misses } & \multicolumn{2}{|l|}{ Adverse events } \\
\hline & Estimated regression coefficient $(95 \% \mathrm{CI})$ & $p$ value & Estimated regression coefficient $(95 \% \mathrm{CI})$ & $p$ value \\
\hline Years of nursing experience & $-0.029(-0.050$ to -0.009$)$ & $<0.01$ & $-0.063(-0.085$ to -0.042$)$ & $<0.001$ \\
\hline \multicolumn{5}{|l|}{ Work place } \\
\hline Internal ward $^{1)}$ & 0.637 (0.170 to 1.104$)$ & $<0.01$ & 0.460 (0.072 to 0.849$)$ & 0.02 \\
\hline Surgical ward ${ }^{1)}$ & $0.147(-0.363$ to 0.657$)$ & 0.57 & $0.139(-0.282$ to 0.561$)$ & 0.52 \\
\hline$E R$ or $I C U^{1)}$ & $0.167(-0.360$ to 0.694$)$ & 0.53 & $-0.097(-0.532$ to 0.337$)$ & 0.66 \\
\hline Number of night shifts per month & 0.077 (0.028 to 0.126$)$ & $<0.01$ & $0.08(0.049$ to 0.111$)$ & $<0.001$ \\
\hline Lack of skill & $0.079(-0.240$ to 0.399$)$ & 0.63 & $0.160(-0.059$ to 0.378$)$ & 0.15 \\
\hline Time pressure & $0.459(0.169$ to 0.749$)$ & $<0.01$ & $0.349(0.121$ to 0.577$)$ & $<0.01$ \\
\hline Lack of communiation ${ }^{2)}$ & $-0.033(-0.378$ to 0.311$)$ & 0.850 & 0.221 (0.003 to 0.440$)$ & 0.04 \\
\hline Nursing stress ${ }^{3)}$ & $0.011(-0.002$ to 0.024$)$ & 0.094 & $0.001(-0.010$ to 0.009$)$ & 0.93 \\
\hline Depression $^{4)}$ & 0.098 (0.059 to 0.136$)$ & $<0.001$ & $-0.010(-0.038$ to 0.017$)$ & 0.46 \\
\hline
\end{tabular}

CI, confidence interval; ER, emergency room; ICU, intensive care unity; SD, standard deviation.

${ }^{1)}$ Calculated in reference to the outpatient department.

${ }^{2)}$ Answered with "no", "occasionally", "frequently", or "always". "No" and "occasionally" were regarded as no, and "frequently" and "always" were regarded as yes.

3) Assesessed using the Nursing Stress Scale.

${ }^{4)}$ Assesessed using the Hospital Anxiety and Depression Scale. 
equation was used with hospitals as the random effects. Age and years of experience were strongly correlated (Spearman's correlation coefficient $=0.88, p<0.001$ ). Since a model using years of experience showed a lower AIC than one using age, we selected a model using years of experience as a potential predictor.

The results of near miss and adverse event analyses were markedly similar. Parameters found to be significantly related to both near misses and adverse events were years of experience, frequency of night shifts, internal ward assignment, and experiencing time pressure. Feelings of depression were significantly related to near misses, whereas no significant relationship was seen between depression and adverse events. A lack of communication between staff was not related to near misses, although a significant relationship was seen between lack of communication and adverse events.

\section{Discussion}

In the present study, we investigated whether or not predictors of near misses and adverse events were similar among nurses at teaching hospitals. Analysis results showed strong similarities between predictors of near misses and adverse events among nurses working at teaching hospitals. These findings suggested a negligible difference between choosing near miss- or adverse event-based analysis when identifying possible causes of adverse events in the medical field.

Given the variation in predictors of near miss and adverse event, we used many variables as potential predictors in the study. As a result, years of experience, frequency of night shifts, assignment to the internal ward, and time pressure were found to be common predictors related to near misses and adverse events, findings which were comparable to those of previous reports.

Several studies have suggested that having fewer years of experience is significantly related to the rate of human error. A lack of experience often results in many stressors and an excessive workload due to a lack of knowledge or administrative ability, while extensive job experience may be related to the ability to detect an error at an early stage and thereby prevent an adverse event $\left.{ }^{15}, 21,31,32\right)$. Night shifts have been implicated in increasing errors due to fatigue and impaired circadian rhythm. Gold et al. conducted a cross-sectional study of nurses working in a large hospital and found that nurses working during the night often felt sleepy, and the frequency of such nurses administering the wrong drug was double compared to those working during the day ${ }^{33)}$. Although a previous study indicated that working in a ward or the ICU is more apt to induce an error than working in the outpatient department ${ }^{32}$, we found that only the internal ward differed significantly from the outpatient department, the reason for which remains unclear. One possible explanation is that the hospitals involved in this study were teaching hospitals. Teaching hospitals typically contain more outpatients than general hospitals, and nurses in the outpatient department often had jobs related to education and research in addition to their routine outpatient department jobs. Such a workload has been associated with the lack of a significant difference observed between the outpatient department, surgical ward, and ER/ICU. Time pressure, likely due to the hectic work environment and lack of staff, has been suggested to reduce attention to work detail and diminish quality of care, thereby potentially inducing errors $^{34)}$.

Several limitations to generalization of results from our study warrant mention. First, in the present study, the numbers of near misses and adverse events were not counted by interview or objective observation, but were instead self-reported on an anonymous basis. Although some measurement bias may have occurred, we chose this approach because incident reporting in hospitals has historically been undervalued ${ }^{35)}$. In contrast, anonymous reporting (particularly with regard to near miss reports) has been found to induce a significant increase in the rate of reported medical errors ${ }^{36)}$, and nonpunitive, confidential voluntary reporting programs provide more useful information regarding errors and their causes than information provided by mandatory reporting programs ${ }^{9,10)}$. Second, a six months period was too long for the participants to recall the number of near misses accurately. However, to avoid period effect, we investigated near misses and adverse events in the same observation period. Although the participants were instructed to check and record the number of the near misses and adverse events during observation period, some recall bias may have occurred. In the present study, the frequencies of near miss and adverse events were relatively low. We suspected that recall bias might have caused the underreporting.

Third, participants were nurses working in teaching hospitals and had younger mean ages than most other hospitals, as is common in Japan. Further, some nurses performed additional jobs unrelated to the routine jobs of nurses working in a general hospital, possibly contributing to selection bias. Fourth, we did not inquire about working hours, which are thought to be strongly related to incident occurrence. However, given data showing that two of the study hospitals had an administrative policy in place to reduce overtime (defined as working more than $40 \mathrm{~h}$ per week) to less than $20 \mathrm{~h}$ per month, we determined that no significant differences 
existed between the hospitals with respect to working hours and that total working hours per month fell between approximately 160 and $180 \mathrm{~h}$. Fifth, we collected no information regarding the type (for example, error of wrong prescription) or degree of near misses and adverse events. Our investigation into frequency and severity of sleepiness experienced at work using a self-report questionnaire may have been unsatisfactory.

In the present study, predictors of near misses and adverse events among nurses working at teaching hospitals were similar. These findings suggested a negligible difference between choosing near miss- or adverse event-based analysis when identifying possible causes of adverse events in the medical field. To generalize our findings, further studies with a wide range of workers in many different fields are necessary.

\section{Acknowledgements}

The work was supported by a Grant-in-Aid (No. 16591159) from the Japan Society for the Promotion of Science.

\section{References}

1) Leape LL (1994) Error in medicine. JAMA 272, 1851-7.

2) Classen DC, Pestotnik SL, Evans RS, Lloyd JF, Burke JP (1997) Adverse drug events in hospitalized patients. Excess length of stay, extra costs, and attributable mortality. JAMA 277, 301-6.

3) Committee on Quality of Health Care in America, institute of medicine (2000) To Err is Human: building a safer Health System, Kohn L, Corrigan J, Donaldson M (Eds.), National Academy Press, Wasington, DC.

4) $\mathrm{Yu} \mathrm{KH}$, Nation RL, Dooley MJ (2005) Multiplicity of medication safety terms, definitions and functional meanings: when is enough enough? Qual Saf Health Care 14, 358-63.

5) Barach P, Small SD (2000) Reporting and preventing medical mishaps: lessons from non-medical near miss reporting systems. BMJ 320, 759-63.

6) Heinrich HW (1980) Industrial accident Prevention. McGraw-Hill, New York.

7) National Resarch Council AoE (1980) Improving aircraft safety: FAA certification of commercial passenger aircraft, Committee on Flight Airworthiness Certification Procedures (Ed.), National Academy of Sciences, Washington, DC.

8) Gambino R, Mallon P (1991) Near misses-an untapped database to find root causes. Lab Report 13, 41-4.

9) Liang BA (1999) Error in medicine: legal impediments to U.S. reform. J Health Polit Policy Law 24, 27-58.

10) Cohen MR (2000) Why error reporting systems should be voluntary. BMJ 320, 728-9.
11) Battles JB, Shea CE (2001) A system of analyzing medical errors to improve GME curricula and programs. Acad Med 76, 125-33.

12) Shojania KG, Wald H, Gross R (2002) Understanding medical error and improving patient safety in the inpatient setting. Med Clin North Am 86, 847-67.

13) Battles JB, Kaplan HS, Van der Schaaf TW, Shea CE (1998) The attributes of medical event-reporting systems: experience with a prototype medical eventreporting system for transfusion medicine. Arch Pathol Lab Med 122, 231-8.

14) Lundy D, Laspina S, Kaplan H, Rabin Fastman B, Lawlor E (2007) Seven hundred and fifty-nine (759) chances to learn: a 3-year pilot project to analyse transfusion-related near-miss events in the Republic of Ireland. Vox Sang 92, 233-41.

15) Phillips J, Beam S, Brinker A, Holquist C, Honig P, Lee LY, Pamer C (2001) Retrospective analysis of mortalities associated with medication errors. Am J Health Syst Pharm 58, 1835-41.

16) Ootim B (2002) Error making. Part 2: identifying the causes in nursing. Nurs Manag (Harrow) 9, 25-9.

17) Committee on the Work Environment for Nurses and Patient Safety (2004) Keeping patients safe: transforming work environment of nurses, Page A (Ed.), National Academy Press, Washington, DC.

18) Chaudhry SI, Olofinboba KA, Krumholz HM (2003) Detection of errors by attending physicians on a general medicine service. J Gen Intern Med 18, 595-600.

19) Sutcliffe KM, Lewton E, Rosenthal MM (2004) Communication failures: an insidious contributor to medical mishaps. Acad Med 79, 186-94.

20) Haw CM, Dickens G, Stubbs J (2005) A review of medication administration errors reported in a large psychiatric hospital in the United kingdom. Psychiatr Serv 56, 1610-3.

21) Tang FI, Sheu SJ, Yu S, Wei IL, Chen CH (2007) Nurses relate the contributing factors involved in medication errors. J Clin Nurs 16, 447-57.

22) Seki Y (2008) Working condition factors associated with time pressure of nurses in Japanese hospitals. J Occup Health 50, 181-90.

23) Hiatt HH, Barnes BA, Brennan TA, Laird NM, Lawthers AG, Leape LL, Localio AR, Newhouse JP, Peterson LM, Thorpe KE (1989) A study of medical injury and medical malpractice. N Engl J Med 321, 480-4.

24) Leape LL, Lawthers AG, Brennan TA, Johnson WG (1993) Preventing medical injury. QRB Qual Rev Bull 19, 144-9.

25) Gray-Toft P, Anderson JA (1981) The nursing stress scale: development of an instrument. J Behav Assess 3, 11-23.

26) Funashima N, Kameoka T, Sugimori M (1997) Reliability and Validity of Japanese version of Role conflict and Ambiguity Scale (RCAS) and that of Nursing Stress Scale (NSS): development 
of Instruments to test the King's Theory of Goal Attainment Chiba Kankaishi 3, 17-23 (in Japanese).

27) French SE, Lenton R, Walters V, Eyles J (2000) An empirical evaluation of an expanded Nursing Stress Scale. J Nurs Meas 8, 161-78.

28) Zigmond AS, Snaith RP (1983) The hospital anxiety and depression scale. Acta Psychiatr Scand 67, 361-70.

29) Bjelland I, Dahl AA, Haug TT, Neckelmann D (2002) The validity of the Hospital Anxiety and Depression Scale. An updated literature review. J Psychosom Res 52, 69-77.

30) Soderberg J, Grankvist K, Brulin C, Wallin O (2009) Incident reporting practices in the preanalytical phase: low reported frequencies in the primary health care setting. Scand J Clin Lab Invest 69, 731-5.

31) Schulmeister L (1999) Chemotherapy medication errors: descriptions, severity, and contributing factors. Oncol Nurs Forum 26, 1033-42.
32) Seki Y, Yamazaki Y (2006) Effects of working conditions on intravenous medication errors in a Japanese hospital. J Nurs Manag 14, 128-39.

33) Gold DR, Rogacz S, Bock N, Tosteson TD, Baum TM, Speizer FE, Czeisler CA (1992) Rotating shift work, sleep, and accidents related to sleepiness in hospital nurses. Am J Public Health 82, 1011-4.

34) Worthington $\mathrm{K}$ (2001) The health risks of mandatory overtime. Am J Nurs 101, 96.

35) Taylor JA, Brownstein D, Christakis DA, Blackburn S, Strandjord TP, Klein EJ, Shafii J (2004) Use of incident reports by physicians and nurses to document medical errors in pediatric patients. Pediatrics 114, 729-35.

36) Taylor JA, Brownstein D, Klein EJ, Strandjord TP (2007) Evaluation of an anonymous system to report medical errors in pediatric inpatients. J Hosp Med 2, 226-33. 